\title{
Case series of abdominal sacral colpopexy
}

\section{Vandana Dhama*, Shakun Singh, Rachna Chaudhary, Haripriya Bajwa}

Department of Obstetrics and Gynecology, LLRM Medical College, Meerut, Uttar Pradesh, India

Received: 20 April 2016

Accepted: 16 May 2016

\section{*Correspondence:}

Dr. Vandana Dhama,

E-mail: vandanallrm@yahoo.com

Copyright: () the author(s), publisher and licensee Medip Academy. This is an open-access article distributed under the terms of the Creative Commons Attribution Non-Commercial License, which permits unrestricted non-commercial use, distribution, and reproduction in any medium, provided the original work is properly cited.

\begin{abstract}
Background: Abdominal sacral colpopexy is a transabdominal procedure that suspends vaginal vault to the sacrum using natural or synthetic graft material. We can perform this procedure in patients who have Post hysterectomy vault prolapse. Primary indication of this procedure is to resuspend a prolapsed vaginal apex. Secondary indications include repair of the cystocele, posterior vaginal wall and apical segment descent. The goal of this study was to assess the complications of repair of post hysterectomy vaginal vault proplase with polypropylene mesh.

Methods: This study was conducted on 8 patients scheduled to undergo abdominal sacral colpopexy in SVBP Hospital associated to LLRM Medical College, Meerut, UP, India from March 2011 to March 2016 who had vault prolapse after hysterectomy. When patients complained of something coming out of vagina, pelvic examination was done by consultant in OPD during valsalva manoeuvre and per speculum examination. Pelvic organ prolapse quantification classification was used to classify prolapse. Two consultants performed all operations based on a standardized surgical technique.

Results: In our study, all patients were followed for up to one year (at 3 months, 6 months, one year) after surgery for following observations- recurrence of prolapse, mesh erosion, dyspareunia, lower abdominal pain, constipation. None of the patient had above complaints in the follow up period.

Conclusions: Abdominal sacral colpopexy with polypropylene mesh is a safe, effective treatment in patients having post hysterectomy vaginal vault proplase. When done by experienced gynecologist, major post-operative complications seem to be very few in patients having normal BMI \& no major systemic illnesses.
\end{abstract}

Keywords: Vaginal vault prolapse, Polypropylene mesh, Abdominal sacral colpopexy

\section{INTRODUCTION}

Abdominal sacral colpopexy was introduced in early 1960 s, it become a widely accepted trans abdominal procedure that suspends the vaginal vault to the sacrum using natural or synthetic grafts. ${ }^{1}$ Abdominal sacral colpopexy attaches the vaginal vault to the sacral promentary and restores the physiological position of the vagina

According to the International continence society, descent of the vaginalapex/ cuff /vaginal vault below a point that is $2 \mathrm{~cm}$ less than the total vaginal length above the plane of hymen is known as vaginal vault proplapse. ${ }^{2}$
Post hysterectomy vault prolapse risk increases with time after hysterectomy and in patients having pelvic organ prolapse previously. ${ }^{3,4}$ Abdominal approach to sacrocolpopexy is associated with a lower incidence of vault prolapse compared to the vaginal sacrospinous fixation. ${ }^{5}$ Studies show success rates of abdominal sacrocolpopexy to be more than $90 \% .^{6,7}$ Primary indication of this procedure is to resuspend a prolapsed vaginal apex. Secondary indications include repair of the anterior vaginal wall (cystocele), posterior vaginal wall and apical segment descent (enterocele and rectocele). vaginal vault proplapse affects quality of women by its local symptoms like bulging, heaviness, pressure ,effects on urinary symptoms like urinary retention and incomplete emptying, bowel symptoms like constipation and sexual functions like dyspareunia. 
Some surgeons use it as a primary procedure along with hysterectomy in patients who have obstructive pulmonary disease, obesity, history of recurrent hernia, or chronic constipation.

Other procedure for vault suspension are sacrospinous ligament fixation and modified uterosacral ligament suspension but sacrocolpopexy has distinct advantages, to maintain length of vagina, axis of vagina and durability due to mesh attachment and less failure rate.

The goal of this study was to assess the complications of repair of post hysterectomy vaginal vault proplase with polypropylene mesh.

\section{METHODS}

This study was conducted on 8 patients admitted to gynaecology ward in SVBP Hospital associated to LLRM Medical College Meerut, from March 2011 to March 2016 who underwent abdominal sacral colpopexy. When a patient came to gynaecology OPD complained of something coming out of vagina or bulge coming out of vagina, she was asked about previous hysterectomy .If answer was affirmative, vault prolapse confirmation was done by per speculum examination and pelvic examination with valsalva manoeuvre.

Inclusion criteria for our study were age $<75$ years, no major systemic illness, only one abdominal procedure previously (hysterectomy), BMI in normal range. Minimum and maximum post hysterectomy interval in our patients was 8.17 years respectively.

Pre-operative investigation like Hemoglobin, packed cell volume, General Blood Picture, Total/Differential Leucocyte count, Blood group, HbA1c, fasting Blood Sugar, Blood urea, Serum creatinine, Urine Routine and Microscopy examination, platelet count, serum bilirubin, ECG and Chest x-ray were done. USG evaluation of lower abdomen was done to rule out any pelvic pathology.

Average age of patients was 56.6 years. Written consent for abdominal sacrocolpopexy was taken from all patients. Risk of recurrence and Mesh erosion also explained to all patients and her relatives. Counselling of all patients and her relatives was done to explain about the procedure \& follow up protocol. Pre-anaesthetic checks up were done for all patients. Bowel preparations was done, prophylactic inj antibiotics like ceftriaxone + sulbactum was given $1 / 2$ hour prior to incision. "Trulene" (Suture India) Nonabsorable PolyPropylene Macroporous Monofilament surgical mesh was used in all patients. Two different gynaecologists (experience more than seven years) performed the operations.

The abdominal sacrocolpopexy was performed by a laparotomy under general anesthesia. Frog leg position was made. Patient was catheterised. A sponge stick was put in the vagina to manipulate the vault. Under full asepsis, Pfannenstiel or vertical incision was given according to the previous incision on the abdomen of the patient. After opening the abdomen, bowels and bladder were packed with laparotomy sponges and retracted by Deaver's retractor.

We held the vault with 3 long allis forceps. We cut the peritoneum on anterior vaginal wall and bladder was separated, similarly we cut peritoneum on posterior vaginal wall. After palpating the sacrum, we cut overlying peritoneum in midline over sacrum and made a tunnel towards the pouch of douglas. After separating loose areolar tissue a shiny white anterior longitudinal ligament was seen. One piece of prolene mesh was attached to anterior vaginal wall and second piece to the posterior vaginal wall (prolene 1-0 suture).

Both mesh were sutured to each other after which the posterior mesh was fixed to the anterior longitudinal ligament on sacrum. The mesh was reperitonealised. Abdomen was closed in layers. An Intra venous fluid was continued for about $24 \mathrm{hrs}$ and intravenous antibiotics for 36 hours. Oral fluids were offered after the bowel sounds were auscultated (on an average after 24 hours). Observation of surgical site- first dressing was done after 48 hours

Urinary catheter was removed after 48 hours. Following definitions were followed to check for the complications in follow up period. Recurrence of prolapse- according to pop q classification, Dyspareunia- painful intercourse, Mesh erosion-presence of exposed graft material, Constipation- infrequent stool, difficult stool passage or both for three month.

\section{RESULTS}

Table 1: Patient profile.

\begin{tabular}{|ll|}
\hline Age (years) & 56.6 \\
\hline Menopausal status & Surgical menopause \\
\hline Incontinence & None \\
\hline & TAH With BSO \\
Type of gynaecological & (4 patients) \\
surgery (previous & TAH \\
operation) & (2 patients) \\
& VH with PFR \\
& (2 patients) \\
\hline BMI $\left(\mathrm{kg} / \mathrm{m}^{2}\right)$ & $22+/-2$ \\
\hline Parity & $>4$ \\
\hline
\end{tabular}

TAH- total abdominal hysterectomy; BSO- bilateral salpingo oophorectomy; BMI- body mass index.

All 8 patients had stage 0 (POP Q classification) in Per Speculum examination at 3 month of follow up. Only one patient had skin dehiscence in post op period. In other patients, post op period was uneventful. 
Table 2: POP Q classification.

\begin{tabular}{|c|c|c|}
\hline \multicolumn{3}{|l|}{ Stages } \\
\hline Stage 0 & \multicolumn{2}{|c|}{ No descent of pelvic structures on straining } \\
\hline Stage 1 & \multicolumn{2}{|c|}{$\begin{array}{l}\text { Leading edge of prolapse }>1 \mathrm{~cm} \text { above } \\
\text { hymen }\end{array}$} \\
\hline Stage 2 & \multicolumn{2}{|c|}{$\begin{array}{l}\text { Leading edge of proplase at level of } \\
\text { introitus, from } 1 \mathrm{~cm} \text { above to } 1 \mathrm{~cm} \text { below the } \\
\text { hymen }\end{array}$} \\
\hline Stage 3 & \multicolumn{2}{|c|}{$\begin{array}{l}\text { Leading edge of proplase }>1 \mathrm{~cm} \text { below } \\
\text { hymen }\end{array}$} \\
\hline Stage 4 & \multicolumn{2}{|c|}{ Complete vaginal vault eversion. } \\
\hline \multicolumn{3}{|c|}{$\begin{array}{l}3 \text { patients had stage } 3 \text { proplapse and } 5 \text { patients had stage } \\
\text { prolapse; In Post-operative assessment, all patients had stage } \\
\text { according to pop Q. }\end{array}$} \\
\hline \multicolumn{3}{|c|}{ Table 3: Surgical complications. } \\
\hline \multicolumn{2}{|c|}{ Operative time } & $150+/-30$ minutes \\
\hline \multicolumn{2}{|c|}{ Estimated blood loss (ml) } & $250+/-50 \mathrm{ml}$ \\
\hline \multicolumn{2}{|c|}{ Hospital stay (days) } & 5+/- 1days \\
\hline \multicolumn{2}{|c|}{$\begin{array}{l}\text { Wound dehiscence (no. of } \\
\text { patient) }\end{array}$} & 1 \\
\hline
\end{tabular}

\section{DISCUSSION}

Abdominal sacral colpopexy with permanent mesh is a safe and effective treatment of the post hysterectomy vaginal vault prolapse. Although some consider this procedure to have long duration of surgery, it has advantage of maintaining long length of vagina and vaginal axis which is not seen with vaginal approach.

Instead of abdominal approach we can suspend vaginal vault to sacrospinous ligament through vaginal approach. Advantage in vaginal approach are short surgical time, short hospital stay but vagina can became narrow, short and axis is also deviated in unilateral sacrospinous fixation. ${ }^{8}$ We can also do laproscopically vaginal vault suspension to the sacrum but it has a long learning curve. Definitely laparoscopic surgery has more advantage over open surgery; less blood loss and short hospital stay are advantages of laparoscopic surgery. ${ }^{9-11}$ In our study, patient were called for follow up after 3 months, 6 months and 12 months of surgery to check for recurrence of proplapse by per speculum examination, urinary symptoms, constipation, dyspareunia, mesh erosion/ mesh extrusion and back pain.

For our study, following complications were observed in follow up of patients-Recurrence of prolapse (according to pop q classification), Dyspareunia (painful intercourse as stated by patient), Mesh erosion (presence of exposed graft material in vagina on PS examination), Constipation (infrequent stool, difficult stool passage or both for three months).

Mesh erosion is one of the complications of this procedure but fortunately no patient came with mesh erosion/ mesh extrusion in follow up period. Incidence of mesh erosion vary 2 to $5 \%$ in different studies. ${ }^{12}$

Wound dehiscence occurred in one patient due poor healing due to old age and due to weakness of tissue. Two units of whole blood also transfused to the same patient. We did her abdominal wound resuturing so her period of stay was extended (12 days). The cause of dehiscence could not be ascertained. So abdominal sacral colpopexy with poly propylene mesh is a safe and effective treatment of the post hysterectomy vaginal vault prolapse.

Limitations of our study were small size of study group, open surgical approach, inexperience of surgeon, pt. having no systemic illness.

Conclusion - abdominal sacral colpopexy with nonabsorbable permanent mesh is a safe and effective treatment of the post hysterectomy vaginal vault proplase.

Funding: No funding sources

Conflict of interest: None declared

Ethical approval: The study was approved by the Institutional Ethics Committee

\section{REFERENCES}

1. Lane FE. Repair of post hysterectomy vaginal-vault prolapse. Obstet Gynecol. 1962;20:72.

2. Haylen BT, de Ridder D, Freeman RM, Swift SE, Berghmans B, Lee $\mathrm{J}$, et al. An International Urogynecological Association (IUGA)/International Continence Society (ICS) joint report on the terminology for female pelvic floor dysfunction. Neurourol Urodyn. 2010;29:4-20.

3. Mant J, Painter R. Epidemiology of genital prolapse: observations from the oxford family planning association study. British Journal of Obstetrics and Gynaecology. 1997;104(5):579-85.

4. Marchionni M, Bracco GL. True incidence of vaginal vault prolapse: thirteen years of experience. Journal of Reproductive Medicine for the Obstetrician and Gynecologist. 1999;44(8):679-84.

5. Paraiso MFR, Walters MD, Rackley RR. Laparoscopic and abdominal sacral colpopexies: a comparative cohort study. American Journal of Obstetrics and Gynecology. 2005;192(5):1752-8.

6. Snyder TE, Krantz KE. Abdominal-retroperitoneal sacral colpopexy for the correction of vaginal prolapse. Obstetrics and Gynecology. 1991;77(6):944-9.

7. Timmons MC, Addison WA. Abdominal sacral colpopexy in 163 women with post hysterectomy vaginal vault prolapse and enterocele: evolution of operative techniques. Journal of Reproductive Medicine for the Obstetrician and Gynecologist. 1992;37(4):323-7.

8. Muhlendorf IK, Browning GM. Vaginal length and sexual function after colpopexy for complete 
uterovaginal eversion. Am J Obstet Gynecol. 1993;169(2 Pt 1):284-7.

9. Velanovich V. Laparoscopic versus open surgery: a preliminary comparison of quality-of-life outcomes. Surg Endosc. 2000;14(1):16-21.

10. Freeman RM, Pantazis K. A randomized controlled trial of abdominal versus laparoscopic sacrocolpopexy for the treatment of posthysterectomy vaginal vault prolapse: LAS study.
International Urogynecology Journal. 2013;24(3):377-84.

11. Klauschie JL, Suozzi BA, O'Brien MM. A comparison of laparoscopic and abdominal sacral colpopexy: objective outcome and perioperative differences. International Urogynecology Journal and Pelvic Floor Dysfunction. 2009;20(3):273-9.

12. Beer M, Kuhn A. Surgical techniques for vault prolapse: a review of the literature. Eur J Obstet Gynecol Reprod Biol. 2005;119:144.

Cite this article as: Dhama V, Singh S, Chaudhary $\mathrm{R}$, Bajwa H. Case series of abdominal sacral colpopexy. Int J Reprod Contracept Obstet Gynecol 2016;5:1992-5. 